Article

\title{
Involvement of MicroRNA-1-FAM83A Axis Dysfunction in the Growth and Motility of Lung Cancer Cells
}

\author{
Pei-Jung Liu ${ }^{1,+}{ }^{+}$, Yu-Hsuan Chen ${ }^{2,+}$, Kuo-Wang Tsai ${ }^{3,+}{ }^{+}$, Hui-Ying Yeah ${ }^{4}$, Chung-Yu Yeh ${ }^{4}$, \\ Ya-Ting $\mathrm{Tu}^{3}$ and Chih-Yun Yang ${ }^{5, *}$ \\ 1 Division of Chest Medicine, Department of Internal Medicine, Kaohsiung Veterans General Hospital, \\ Kaohsiung 81362, Taiwan; pjliu20130201@gmail.com \\ 2 Division of Chest Medicine, Department of Internal Medicine, CHENG HSIN General Hospital, Taipei 11221, \\ Taiwan; anemia0829@gmail.com \\ 3 Department of Research, Taipei Tzu Chi Hospital, Buddhist Tzu Chi Medical Foundation, New Taipei 23124, \\ Taiwan; kwtsai6733@gmail.com (K.-W.T.); new0224@hotmail.com (Y.-T.T.) \\ 4 Department of Medical Education and Research, Kaohsiung Veterans General Hospital, Kaohsiung 81362, \\ Taiwan; hyyeh@vghks.gov.tw (H.-Y.Y.); cyyeah@vghks.gov.tw (C.-Y.Y.) \\ 5 Division of Chest Medicine, Kaohsiung Municipal Min-Sheng Hospital, Kaohsiung 802213, Taiwan \\ * Correspondence: chihyunyang@gmail.com \\ + These authors contributed equally to this work.
}

Received: 9 November 2020; Accepted: 20 November 2020; Published: 22 November 2020

\begin{abstract}
Lung cancer is the most prevalent types of cancer and the leading cause of cancer-related deaths worldwide. Among all cancers, lung cancer has the highest incidence, accompanied by a high mortality rate at the advanced stage. Favorable prognostic biomarkers can effectively increase the survival rate in lung cancer. Our results revealed FAM83A (Family with sequence similarity 83, member A) overexpression in lung cancer tissues compared with adjacent normal tissues. Furthermore, high FAM83A expression was closely associated with poor lung cancer survival. Here, through siRNA transfection, we effectively inhibited FAM83A expression in the lung cancer cell lines H1355 and A549. FAM83A knockdown significantly suppressed the proliferation, migration, and invasion ability of these cells. Furthermore, FAM83A knockdown could suppress Epidermal growth factor receptor (EGFR)/Mitogen-activated protein kinase (MAPK)/Choline kinase alpha (CHKA) signaling activation in A549 and H1355. By using a bioinformatics approach, we found that FAM83A overexpression in lung cancer may result from miR-1-3p downregulation. In summary, we identified a novel miR-1-FAM83A axis could partially modulate the EGFR/choline phospholipid metabolism signaling pathway, which suppressed lung cancer growth and motility. Our findings provide new insights for the development of lung cancer therapeutics.
\end{abstract}

Keywords: lung cancer; FAM83A; miR-1-3p

\section{Introduction}

Lung cancer is the most common cancer and the leading cause of cancer-related deaths worldwide. According to histological features and appearance of malignant cells, lung cancer is classified into 2 types: non-small-cell lung cancer (NSCLC) and small-cell lung cancer. Of them, NSCLC is more common, accounting for approximately $85 \%$ of all lung cancers. More than $40 \%$ of NSCLC cases are metastatic at diagnosis [1]. The primary subtypes of NSCLC include lung adenocarcinoma (LUAD), lung squamous cell carcinoma (LUSC), and large-cell carcinoma [2]. Over the past decade, the treatment of NSCLC and development of therapeutic approaches have improved considerably [3]. However, 
the mortality rate of patients with advanced lung cancer remains high. The development of reliable diagnostics and identification of prognostic biomarkers are essential to increasing the survival rate of patients with lung cancer.

Lung cancer progression depends on the effects of individual oncogenes; therefore, oncogene-targeted therapy has been confirmed to be effective. However, cancer cells can also be adapted by compensatory activation of the downstream pathway and develop resistance to target therapy. Family with sequence similarity 83 , member A (FAM $83 A)$ is a gene widely expressed in eukaryotic cells, particularly in the epithelial cells of the skin, bladder, kidneys, and lungs [4]. FAM83A is highly expressed in several human cancers, including pancreatic, breast, and lung cancer and hepatocellular carcinoma [5-11]. It plays an oncogenic role in promoting cancer cell proliferation and metastasis through the mitogen-activated protein kinase (MAPK)/extracellular signal-regulated kinase and phosphatidylinositol-3-kinase (PI3K)/protein kinase B (AKT)/mammalian target of rapamycin pathways [9,12-17]. However, FAM83A overexpression mechanism in human cancer and its potential effects on lung cancer have yet to be established. In this study, we assessed the effects of FAM83A on lung cancer and reported for the first time that microRNA participates in FAM $83 A$ overexpression in lung cancer.

\section{Results}

\subsection{Identification of Prognostic Biomarkers for Lung Cancer}

We identified genes whose dysfunction is involved in lung cancer progression. Data on a total of 511 RNA transcriptomes and the clinical data of patients with LUAD were downloaded from The Cancer Genome Atlas (TCGA) database. Analysis of the correlations between the clinical characteristics and overall survival of patients with LUAD revealed that the advanced stage was associated with poor survival (crude hazard ratio (cHR) 2.76, 95\% CI 2.00-3.80, $p<0.001$; Table 1). We further identified differentially expressed genes between patients with stage I + II and stage III + IV lung cancer. The genes were selected according to the following criteria: FPKM $>1$, fold change of $>1.5$ or $<0.75$, and $p<0.05$ (Figure 1). Compared with those with stage I + II lung cancer, patients with stage III + IV lung cancer demonstrated significantly increased expression of 12 candidate genes but significantly decreased expression of 26 candidate genes (Table S1). As Figure 1B shows, FAM83A expression was significantly upregulated in LUAD tissues compared with in adjacent normal tissues. FAM83A expression was also significantly increased in patients with advanced lung cancer, large tumor size, and lymph node metastasis (Table 2). As Figure 2 shows, FAM83A expression was significantly elevated in patients with advanced lung cancer $(p=0.02$ and $p<0.001$ for stage I vs. stages II and III + IV, respectively), lymph node metastasis ( $p=0.03$ and $p<0.001$ for N0 vs. N1 and $>\mathrm{N} 2$, respectively) and large tumor size ( $p<0.001$ and $p<0.001$ for T1 vs. T2 and T3 + T4, respectively). FAM83A expression was also significantly increased in LUSC tissues compared with in adjacent normal tissues $(p<0.001)$ (Figure S1A). However, no correlation between FAM83A expression and the clinicopathological features of patients with LUSC was noted (Figure S1 and Table S2). 
(A)

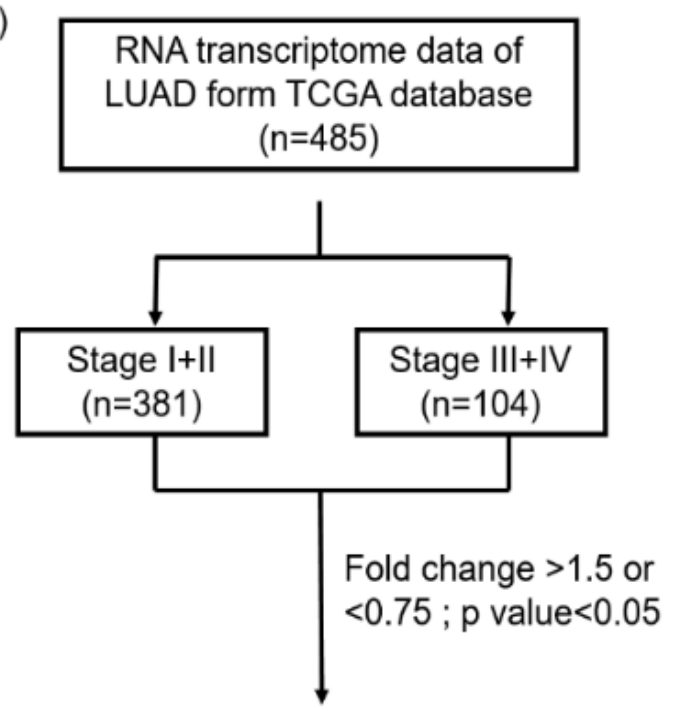

\section{Up regulation: 12 Candidates}

Down regulation: 26 Candidates
(B)

\section{FAM83A}

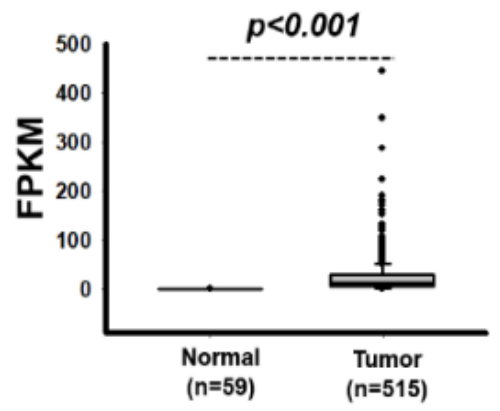

Figure 1. Identification of candidate genes associated with stage in lung adenocarcinoma (LUAD) through TCGA database analysis. (A) Flowchart of the identification process. (B) Increased FAM83A expression in LUAD tissues compared with that in adjacent normal tissues.

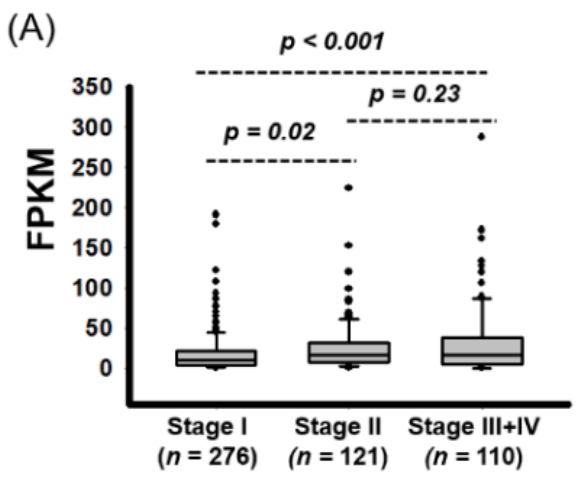

(C)

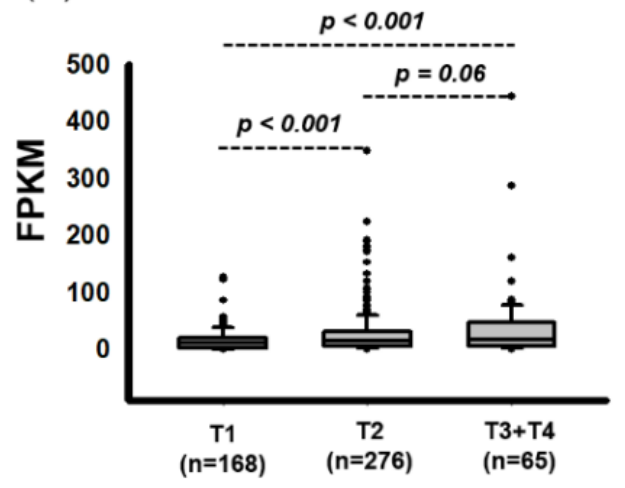

(B)

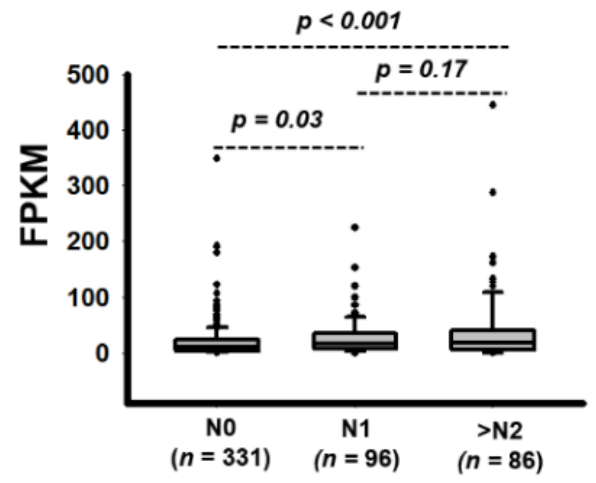

(D)

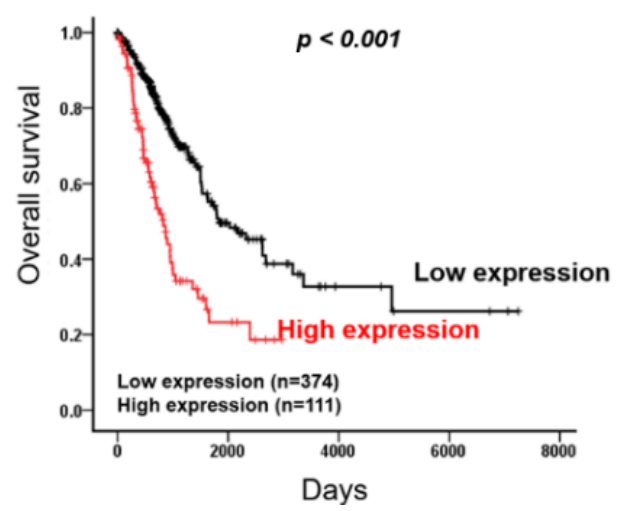

Figure 2. Association of $F A M 83 A$ Expression with Poor LUAD Prognosis. (A) FAM83A expression in LUAD by pathological stage. (B) FAM83A expression in LUAD by lymph node metastasis status. (C) FAM83A expression in LUAD by T stage. (D) Kaplan-Meier survival curves showing the effect of FAM83A expression on overall survival in LUAD. 
Table 1. Univariate Cox's regression analysis of clinicopathological characteristics for overall survival of 485 patients with lung adenocarcinoma (LUAD).

\begin{tabular}{cccc}
\hline \multirow{2}{*}{ Characteristic } & No. (\%) & \multicolumn{2}{c}{ OS } \\
\cline { 3 - 4 } & & CHR (95\% CI) & $p$-Value \\
\hline Pathology stage & & 1.00 & \\
\hline I + II & $381(78.6)$ & $2.76(2.00-3.80)$ & $<0.001$ \\
III + IV & $104(21.4)$ & & \\
\hline pT stage $(n=482)$ & & 1.00 & $<0.001$ \\
\hline T1 + T2 & $423(87.2)$ & $2.43(1.65-3.60)$ & $<0.001$ \\
T3 + T4 & $62(12.8)$ & & $<0.001$ \\
\hline pN stage $(n=481)$ & & $2.49(1.73-3.58)$ & \\
\hline N0 & $323(66.6)$ & $3.31(2.24-4.88)$ & \\
N1 & $89(18.4)$ & & 0.003 \\
\hline N2 & $73(15.0)$ & 1.00 & \\
\hline pM stage & & $2.23(1.31-3.80)$ & \\
\hline M0 & $460(94.8)$ & & \\
M1 & $25(5.2)$ & & \\
\hline
\end{tabular}

Abbreviation:.OS, overall survival; CHR, crude hazard ratio.

Table 2. Correlation of $F A M 83 A$ expression with clinicopathological characteristics of lung adenocarcinoma patients (LUAD).

\begin{tabular}{|c|c|c|c|c|}
\hline \multirow{2}{*}{ Variables } & \multicolumn{4}{|c|}{ FAM83A $(n=511)$} \\
\hline & No. $(\%)$ & Mean \pm SD & Median & $p$-Value \\
\hline \multicolumn{5}{|c|}{ Pathology stage } \\
\hline I & $275(53.8)$ & $18.05 \pm 25.58^{\mathrm{cd}}$ & 10.32 & \multirow[t]{3}{*}{$<0.001^{\mathrm{a}}$} \\
\hline II & $126(24.7)$ & $24.28 \pm 30.47^{c}$ & 16.16 & \\
\hline III+ IV & $110(21.5)$ & $41.67 \pm 66.33^{d}$ & 20.54 & \\
\hline \multicolumn{5}{|c|}{ pT stage $(n=508)$} \\
\hline $\mathrm{T} 1$ & $169(33.1)$ & $15.29 \pm 18.70$ ef & 10.81 & \multirow[t]{3}{*}{$<0.001^{\mathrm{a}}$} \\
\hline $\mathrm{T} 2$ & $276(54.0)$ & $26.81 \pm 39.51^{\mathrm{e}}$ & 14.64 & \\
\hline $\mathrm{T} 3+\mathrm{T} 4$ & $66(12.9)$ & $39.07 \pm 67.64^{\mathrm{f}}$ & 17.20 & \\
\hline \multicolumn{5}{|c|}{ pN stage $(n=507)$} \\
\hline No & $339(66.3)$ & $19.33 \pm 30.56 \mathrm{gh}$ & 11.00 & \multirow[t]{3}{*}{$<0.001^{\mathrm{a}}$} \\
\hline N1 & $95(18.6)$ & $27.61 \pm 34.32^{g}$ & 16.85 & \\
\hline$>\mathrm{N} 2$ & $77(15.1)$ & $43.91 \pm 67.96^{h}$ & 21.45 & \\
\hline \multicolumn{5}{|l|}{ pM stage } \\
\hline M0 & 485 (94.9) & $23.09 \pm 32.62$ & 12.72 & \multirow[t]{2}{*}{$0.531^{b}$} \\
\hline M1 & $26(5.1)$ & $54.10 \pm 105.81$ & 18.65 & \\
\hline
\end{tabular}

a $p$-values were estimated by Kruskal-Wallis 1-way ANOVA test. ${ }^{\mathrm{b}} p$-value were estimated by Mann-Whitney U test. ${ }^{\mathrm{c}} p=0.003,{ }^{\mathrm{d}} p<0.001,{ }^{\mathrm{e}} p<0.001,{ }^{\mathrm{f}} p<0.001, \mathrm{~g} p=0.001,{ }^{\mathrm{h}} p<0.001$.

\subsection{High FAM83A Expression Correlated with Worse Survival in Patients with LUAD}

We assessed the relationship between FAM83A expression and postoperative survival of patients with lung cancer. Firstly, we defined a cutoff value for $F A M 83 A$ expression levels, which was determined using receiver operating characteristic analysis. Patients with LUAD were separated into two groups representing high and low FAM83A expression on the basis of this cutoff value (30.4). The Kaplan-Meier survival curves showed that compared with low levels, high expression levels of FAM83A were closely associated with shorter survival duration (Figure 2D). Univariate Cox regression analysis revealed that 
high expression of FAM83A was correlated with poor survival (cHR 2.57, 95\% CI 1.87-3.54, $p<0.001$; Table 3). Multivariate Cox regression analysis found high FAM83A expression to be an independent prognostic biomarker for overall survival in LUAD (adjusted hazard ratio (aHR) 2.17, 95\% CI 1.57-3.01, $p<0.001$; Table 3). Based on the defined cutoff values (19.6), a significant correlation between high FAM83A expression and poor survival in LUSC was demonstrated in both univariate (cHR 1.52, 95\% CI 1.14-2.05, $p=0.005$ ) and multivariate analysis (aHR 1.49, 95\% CI 1.11-2.00, $p=0.008$; Figure S2 and Table S3). In summary, FAM83A may be an independent prognostic biomarker for overall survival in lung cancer.

Table 3. Univariate and multivariate Cox's regression analysis of gene expression for overall survival of 485 patients with lung cancer.

\begin{tabular}{cccccc}
\hline \multirow{2}{*}{ Characteristic } & No. (\%) & \multicolumn{5}{c}{ OS } \\
\cline { 3 - 6 } & & CHR (95\% CI) & $p$-Value & AHR (95\% CI) & $p$-Value \\
\hline FAM83A & $(n=485)$ & & & 1.00 & \\
\hline Low & $374(77.1)$ & 1.00 & $2.17(1.57-3.01)$ & $<0.001$ \\
\hline High & $111(22.9)$ & $2.57(1.87-3.54)$ & $<0.001$ & & \\
\hline
\end{tabular}

OS, Overall survival; CHR, crude hazard ratio; AHR, adjusted hazard ratio. AHR were adjusted for AJCC pathological stage (II, III and IV vs. I).

\subsection{FAM83A Regulates Lung Cancer Cell Growth and Motility}

To explore the role of FAM83A in lung cancer, we assessed its effects on lung cancer cell growth. We knocked down its expression in $\mathrm{H} 1355$ and A549 cells through transfection of siRNAs, revealing that expression levels of FAM83A could be inhibited by siFAM83A\#1 or siFAM83A \#2 transfection (Figure $3 \mathrm{~A}, \mathrm{~B})$. FAM83A knockdown substantially suppressed cell proliferation and colony formation ability in both cell lines (Figure $3 \mathrm{C}-\mathrm{H}$ ). The expression levels of cyclin B and D, which are associated with the cell cycle in lung cancer, were reduced whereas that of p27 was increased in the A549 and H1355 cells (Figure 3I,J). FAM83A knockdown substantially inhibited the migration and invasion ability of both the A549 and H1355 cells (Figure 4A-F).

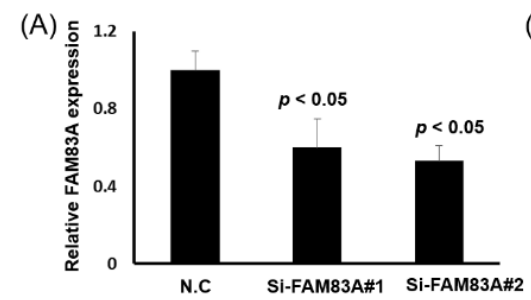

(C)

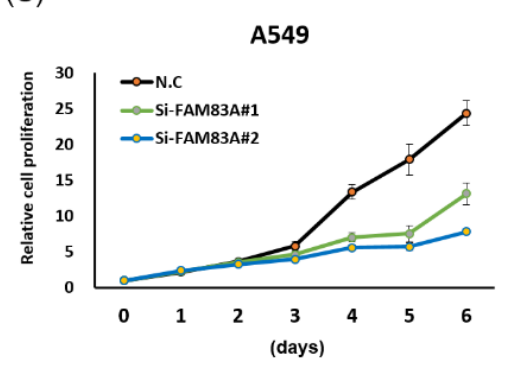

(B)

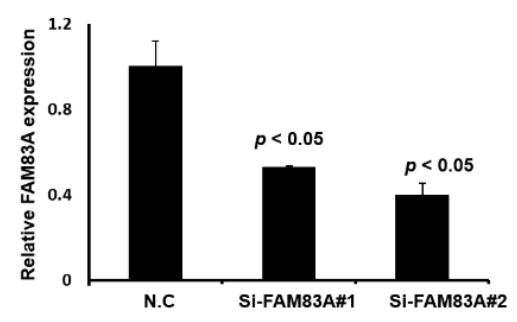

(D)

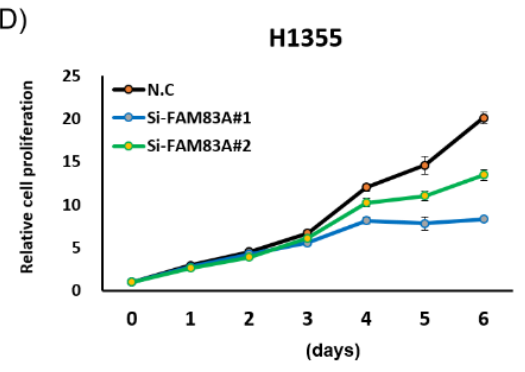

Figure 3. Cont. 
(E)

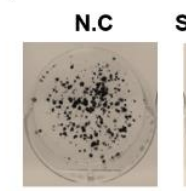

(G)

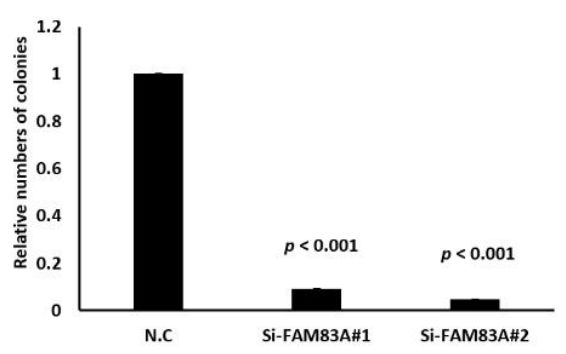

(I)

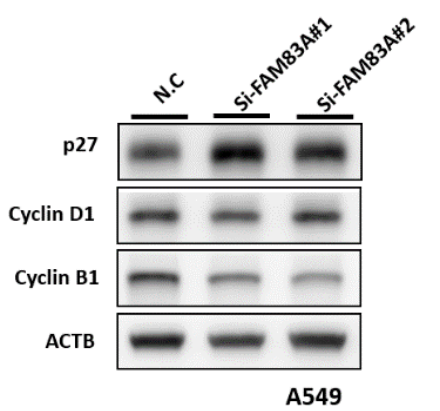

( $\mathrm{F})$

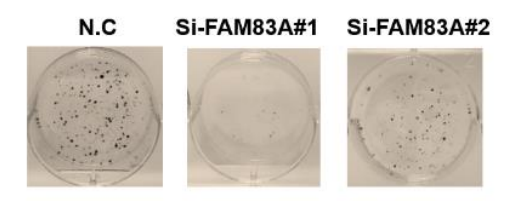

(H)

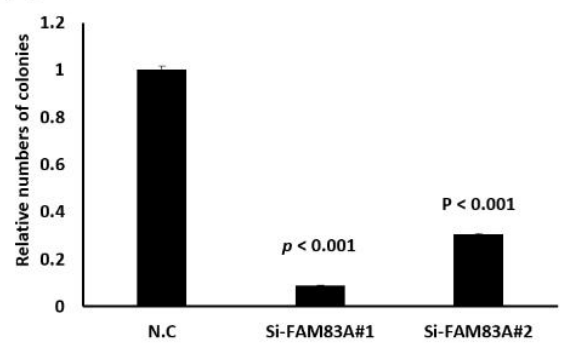

$(\mathrm{J})$

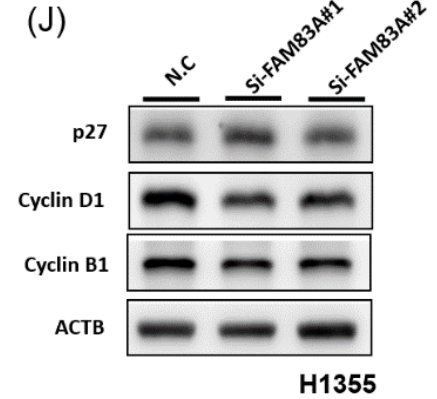

Figure 3. Suppression of lung cancer cell growth and motility by FAM83A knockdown. (A,B) FAM83A expression levels in A549 and H1355 cells knocked down using siRNA transfection by real-time reverse transcription polymerase chain reaction. (C,D) Cell proliferation in A549 and H1355 cells with FAM83A knockdown. (E-H) Quantification of colony formation ability of the A549 and H1355 cells. (I,J) Western blot analysis of expression levels of cell cycle-related genes in the A549 and H1355 cells. N.C. indicates a random siRNA sequence control. All experiments were performed in triplicate, and the data were analyzed using Student's $t$ test. Differences were considered significant when $p<0.05$.

(A)

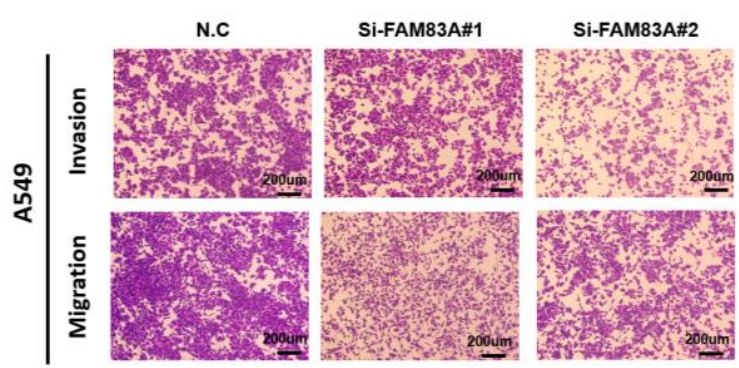

(B)

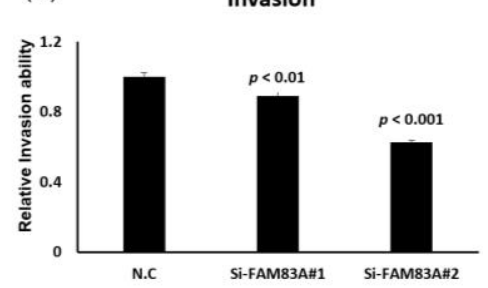

(C)

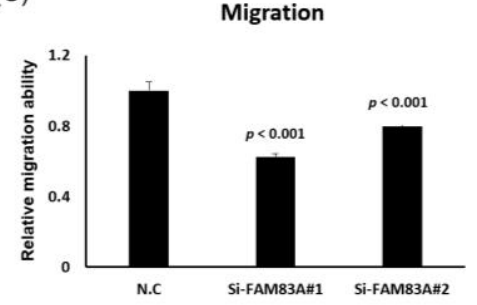

Figure 4. Cont. 
(D)

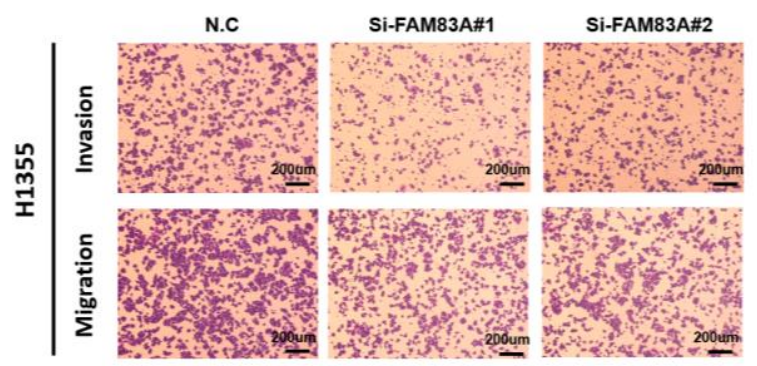

$(\mathrm{E})$

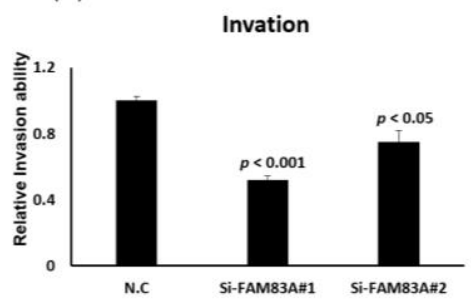

(F)

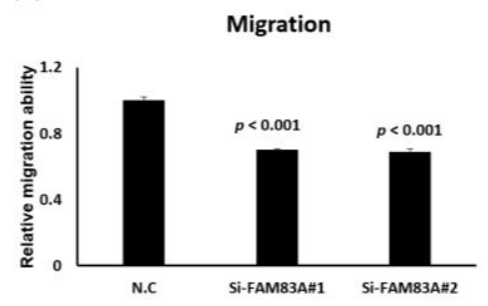

Figure 4. Suppression of lung cancer cell motility by FAM83A knockdown. (A,B) Cell invasion and migration in A549 cells with FAM83A knockdown. (C) Quantification of invading and migrating A549 cells. (D) Cell invasion and migration in H1355 cells with FAM83A knockdown. (E,F) Quantification of invading and migrating H1355 cells. N.C. indicates a random siRNA sequence control. All experiments were performed in triplicate, and the data were analyzed using Student's $t$ test. Differences were considered significant when $p<0.05$. scale bar $=200 \mu \mathrm{m}$.

\subsection{Identification of FAM83A-Associated Signaling Pathways}

To assess the mechanism of FAM83A knockdown-induced inhibition of growth and invasion ability of lung cancer cells, we performed transcriptome profiling using a microarray approach. The biological function of FAM83A knockdown is very similar in A549 and H1355 cells. Therefore, we only performed gene expression profiles on A549 cells with FAM83A knockdown. From the microarray data, we identified the putative signaling pathways in FAM83A that suppress lung cancer growth and motility. We analyzed 4 transcriptome profiles: those of 2 controls and siFAM83A\#1 and siFAM83A\#2 with FAM83A knockdown. We analyzed the sample diversity using principal coordinate analysis. As Figure S3A shows, the gene expression pattern of the 2 controls were similar, and those of siFAM83A \#1 and siFAM83A \#2 were distinct from the control groups. We further identified differentially expressed genes in A549 cells with FAM83A knockdown: 335 that were significantly upregulated (>1.5-fold) and 69 that were significantly downregulated (Figure S3B). Pathway enrichment analysis revealed that these 404 genes are critically involved in 16 signaling transduction pathways: Alzheimer disease, thermogenesis, choline metabolism in cancer, renin secretion, Parkinson disease, oxidative phosphorylation, gap junction, glutamatergic synapse, cortisol synthesis and secretion, long-term potentiation, degradation of valine, leucine, and isoleucine, endocytosis, complement and coagulation cascades, propanoate metabolism, legionellosis, and the phospholipase D signaling pathway (Figure S3C). We deduced that choline metabolism signaling may be involved in FAM83A knockdown-induced inhibition of growth and motility ability of lung cancer cells. Therefore, we examined the Epidermal growth factor receptor (EGFR)/MAPK/Choline kinase alpha (CHKA) signaling activity in A549 and H1355 cells further with FAM83A knockdown. As illustrated in Figure 5A,B, the expression levels of EGFR and CHKA, and the phosphorylation of EGFR and MAPK were reduced in lung cancer cells with FAM83A knockdown. These results indicated that FAM83A knockdown-induced inhibition of lung cancer cell growth and motility was partially caused by EGFR/MAPK/CHKA signaling activity suppression. 
EGF treatment

(A)

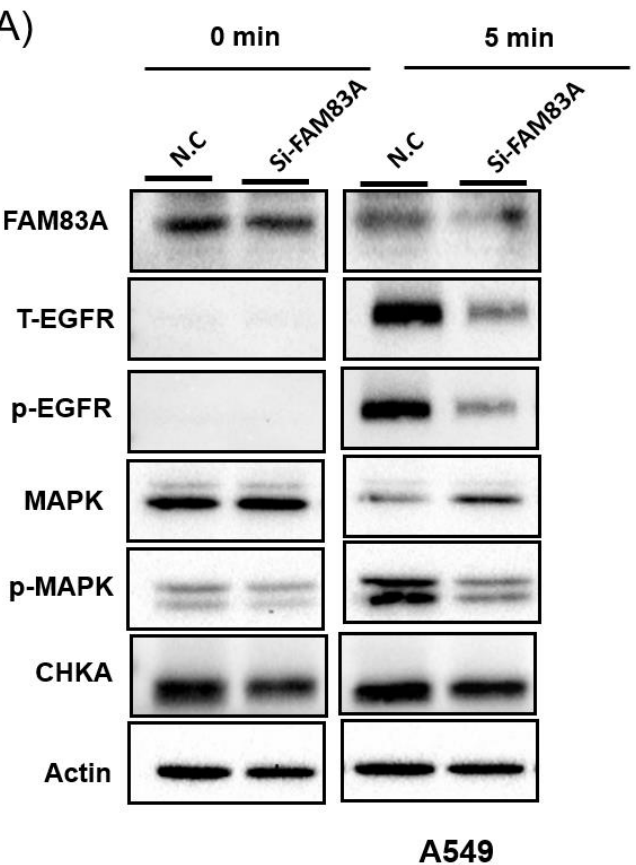

EGF treatment

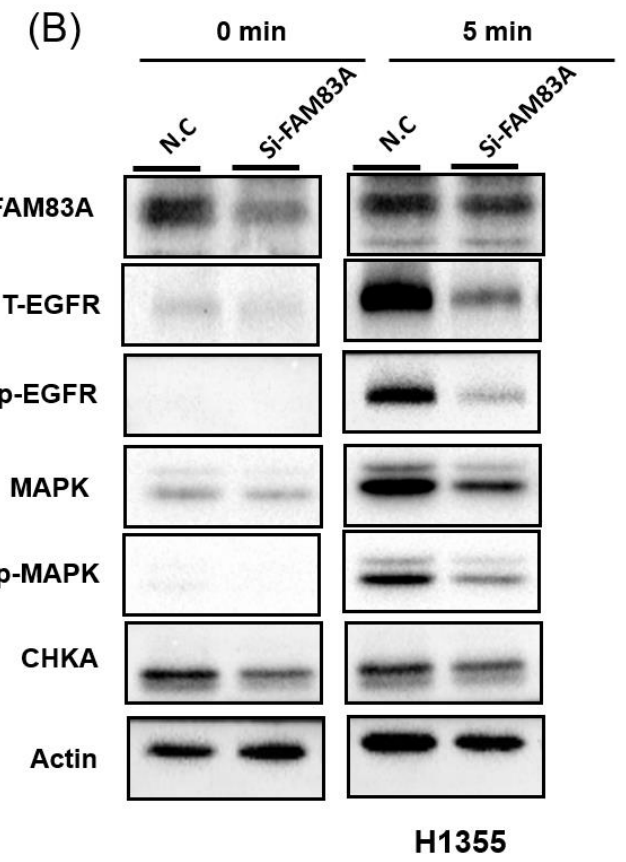

Figure 5. FAM $83 A$ knockdown-induced inhibition of lung cancer cell growth and motility by choline metabolism signaling. After 5 min of Epidermal growth factor (EGF) treatment, the expression levels of Epidermal growth factor receptor (EGFR), Phosphorylation-EGFR (p-EGFR), Mitogen-activated protein kinae (MAPK), Phosphorylation-MAPK (p-MAPK), and Choline kinase alpha (CHKA) in (A) A549 and (B) H1355 cells with FAM83A knockdown were assessed using Western blot assay. N.C. indicates a random siRNA sequence control. All experiments were performed in triplicate, and the data were analyzed using Student's $t$ test. Differences were considered significant when $p<0.05$.

\section{5. miR-1-3p Suppresses FAM83A Expression by Targeting the 3 Prime Untranslated Region of FAM83A}

Our data revealed high FAM83A expression in LUAD tissues and its contribution to disease progression. However, the mechanisms underlying abnormal FAM83A expression remain unclear. MicroRNAs are small nuclear noncoding RNAs that suppress gene translation or promote mRNA degradation by binding to the $3^{\prime}$ untranslated region ( $3^{\prime} \mathrm{UTR}$ ). We attempted to identify the miRNAs that regulate FAM $83 A$ expression in lung cancer. The microRNA.org prediction tool selected 13 miRNA candidates expected to bind to the $3^{\prime} \mathrm{UTR}$ of FAM83A. We then examined the expression of these miRNAs in LUAD by analyzing TCGA database (Figure 6A). As miRNAs suppress target gene expression, we expected them to interact directly with the $3^{\prime}$ UTR of FAM83A and that FAM83A expression would be negatively correlated with target genes in LUAD. We found that miR-1 regulated FAM83A expression by targeting its $3^{\prime} \mathrm{UTR}$. Moreover, miR-1 expression was both negatively correlated with FAM83A expression and significantly reduced in LUAD (Figure 6B,C). Low miR-1-3p expression was significantly correlated with advanced stage and worse overall survival in LUAD (Figure 6D and Tables 4 and 5).

Furthermore, both FAM $83 A$ mRNA and protein expression levels in lung cancer cells was considerably reduced after $m i R-1$ mimic transfection (Figure 7A-C). We cloned the $3^{\prime} \mathrm{UTR}$ sequence into the pmiR-REPORT Luciferase miRNA Expression Reporter Vector System. As Figure 7D,E shows, luciferase activity was significantly reduced in A549 cells after miR-1-3p cotransfection. When the miR-1-3p binding sites were mutated, this suppression was eliminated (Figure 7F), indicating that miR-1-3p inhibited FAM83A expression by directly binding to its $3^{\prime} \mathrm{UTR}$. To assess the effects of miR-1-3p-FAM83A axis activity on LUAD, we classified the combined expression of FAM83A and miR-1-3p into 3 groups: high FAM83A and low miR-1-3p, low or high levels of both FAM83A and 
miR-1-3p, and low FAM83A and high miR-1-3p. As Figure 7G shows, high FAM83A and low miR-1-3p expression were significantly associated with poor survival in patients with LUAD.

(A)

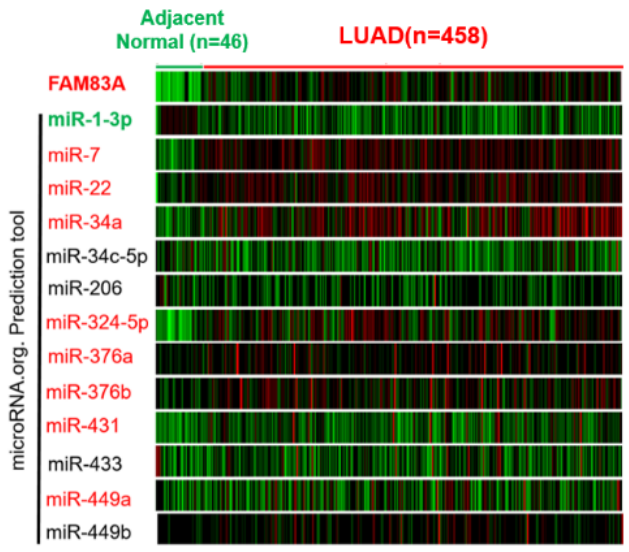

(B)

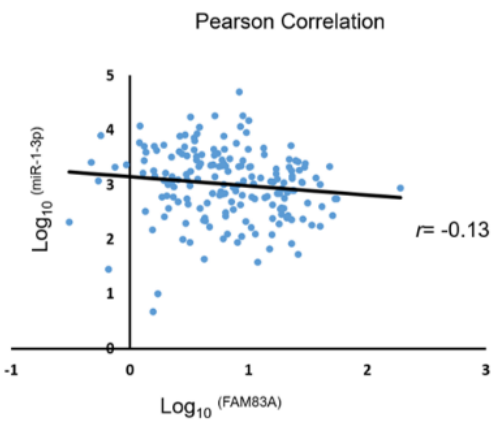

(C)

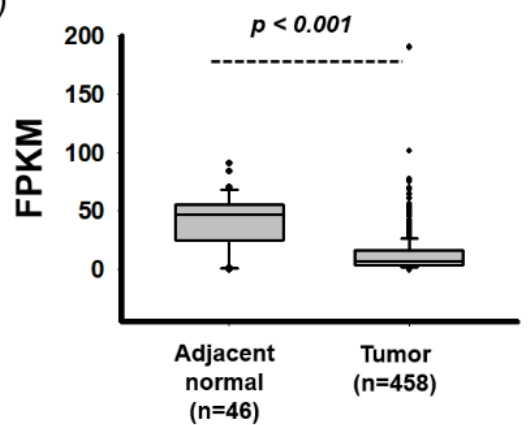

(D)

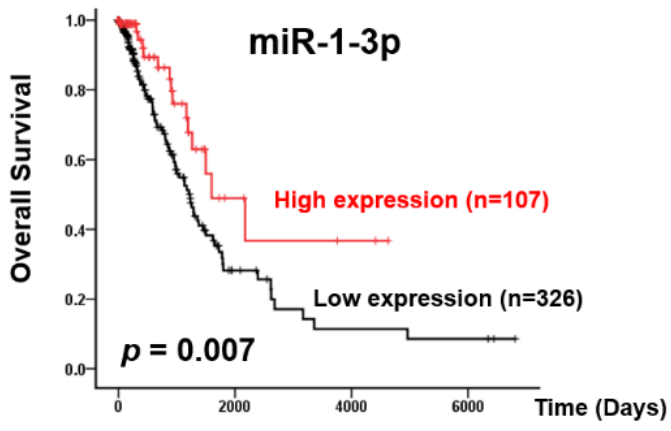

Figure 6. Identification of putative miRNA candidates for FAM $83 A$ knockdown through a bioinformatics approach. (A) Identification of miRNA candidates to regulate FAM83A expression. Expression levels of the 13 miRNAs and FAM83A in 458 LUAD and 46 normal tissues are shown as heat maps. Candidate genes whose expression was significantly increased $(p<0.01)$ or reduced in lung cancer tissues compared with in adjacent normal tissues are labeled in red and green, respectively. (B) Pearson correlation analysis of miR-1-3p and FAM83A expression in 185 patients with LUAD. (C) TCGA database analysis of miR-1-3p expression in lung cancer. (D) Kaplan-Meier survival curves showing the effects of miR-1-3p expression on overall survival of patients with LUAD.

(A)

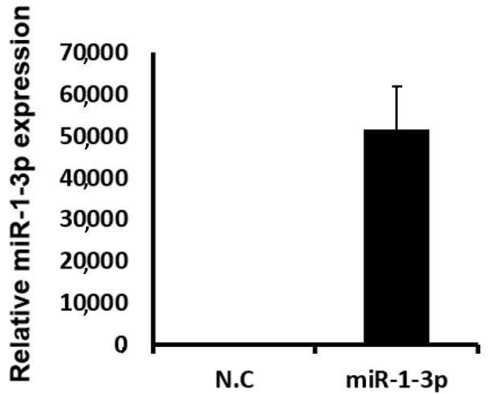

(B)

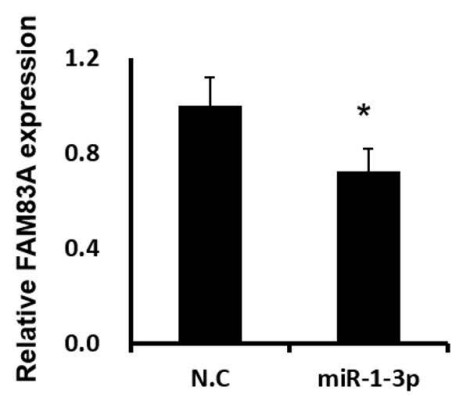

Figure 7. Cont.
(C)

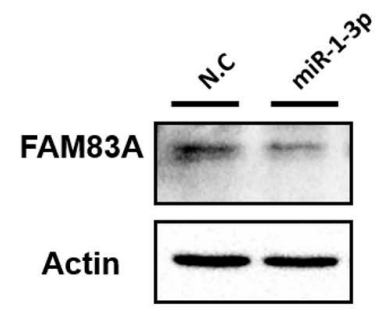


(D)

\author{
5' tg-ACcTG--TgCagcACATTCCa 3' FAM83A-3'UTR (WT) \\ | | |: | | | || || \\ 3' uaUGuAUgAaGaaaUGUAAGGu 5' has-miR-1-3p \\ 5' tg-TGcAC--AgGagcTGTAAGGa 3' FAM83A-3'UTR (Mut)
}

$(\mathrm{E})$

FAM83A-3'UTR WT

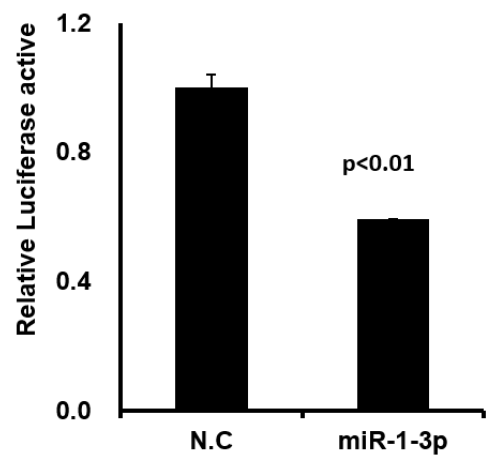

$(\mathrm{F})$

\author{
FAM83A-3'UTR Mut
}

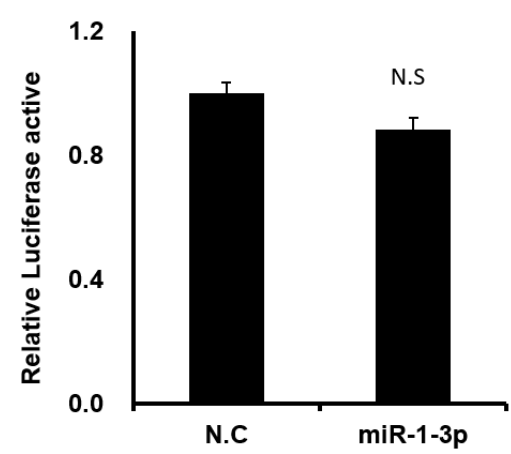

(G)

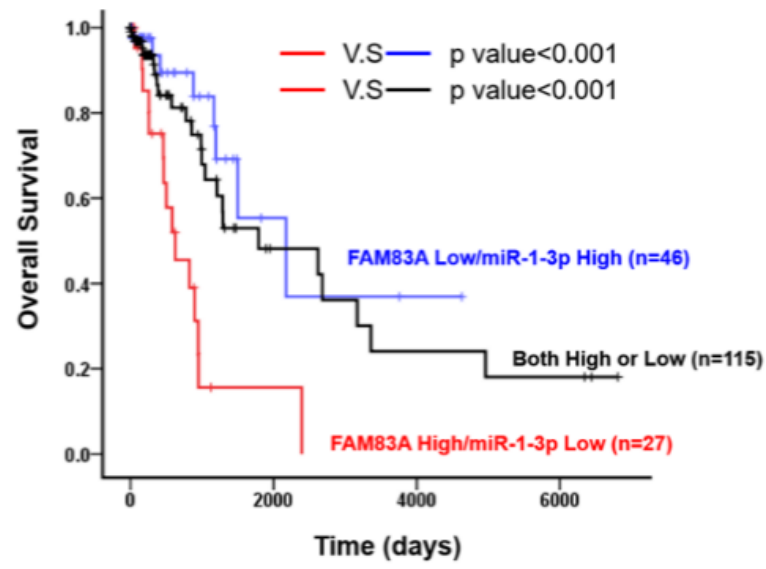

Figure 7. Suppression of $F A M 83 A$ expression by miR-1-3p through direct binding to the $3^{\prime}$ untranslated region ( $3^{\prime}$ UTR) of FAM83A. (A) miR-1-3p expression in lung cancer cells with miR-1-3p mimic transfection. (B) FAM83A mRNA expression in lung cancer cells with miR-1-3p and negative control transfection. (C) FAM83A protein expression in lung cancer cells with miR-1-3p and negative control transfection. (D-F) Reporter construct with wild-type FAM83A 3'UTR or binding site mutant after miR-1-3p cotransfection into lung cancer cells, followed by assessment of luciferase activity. (G) Effects of combined FAM $83 A$ and miR-1-3p expression on overall survival in lung cancer. N.C. indicates a random siRNA sequence control. All experiments were performed in triplicate, and the data were analyzed using Student's $t$ test. Differences were considered significant when ${ }^{*} p<0.05$. 
Table 4. Correlation of miR-1-3p expression with clinicopathological characteristics of 453 lung cancer patients.

\begin{tabular}{|c|c|c|c|c|}
\hline \multirow{2}{*}{ Variables } & \multicolumn{4}{|c|}{$\operatorname{miR}-1-3 p(n=453)$} \\
\hline & No. (\%) & Mean \pm SD & Median & $p$-Value \\
\hline \multicolumn{5}{|c|}{ Pathology stage } \\
\hline I & $248(54.7)$ & $13.24 \pm 18.01$ & 8.07 & $0.325^{\mathrm{a}}$ \\
\hline II & $115(25.4)$ & $10.18 \pm 12.27$ & 5.19 & \\
\hline III & $73(16.1)$ & $10.88 \pm 10.13$ & 6.49 & \\
\hline IV & $17(3.8)$ & $12.71 \pm 20.41$ & 6.13 & \\
\hline \multicolumn{5}{|l|}{ pT stage } \\
\hline $\mathrm{T} 1$ & 157 (34.7) & $15.18 \pm 16.56^{\mathrm{e}}$ & 10.01 & $0.002^{b}$ \\
\hline $\mathrm{T} 2$ & 238 (52.5) & $10.02 \pm 15.63^{\mathrm{e}}$ & 5.95 & \\
\hline T3 & $43(9.5)$ & $12.68 \pm 13.92$ & 6.55 & \\
\hline $\mathrm{T} 4$ & $15(3.3)$ & $10.20 \pm 7.87$ & 6.61 & \\
\hline \multicolumn{5}{|l|}{ pN stage } \\
\hline No & $306(67.5)$ & $12.53 \pm 17.29$ & 6.74 & $0.772^{\mathrm{a}}$ \\
\hline N1 & $81(17.9)$ & $11.46 \pm 13.12$ & 7.67 & \\
\hline N2 & $65(14.3)$ & $10.80 \pm 10.48$ & 6.49 & \\
\hline N3 & $1(0.3)$ & $3.03 \pm$ & 3.03 & \\
\hline \multicolumn{5}{|l|}{ pM stage } \\
\hline M0 & $436(96.2)$ & $12.04 \pm 15.58$ & 6.74 & $0.863^{c}$ \\
\hline M1 & $17(3.8)$ & $12.71 \pm 20.41$ & 6.13 & \\
\hline
\end{tabular}

a $p$-value were estimated by one-way ANOVA test. ${ }^{\mathrm{b}} p$-values were estimated by Kruskal-Wallis 1-way ANOVA test. c $p$-value were estimated by Student's $t$ test, ${ }^{\mathrm{e}} p<0.001$.

Table 5. Univariate and multivariate Cox's regression analysis of miR-1-3p expression for overall survival of 433 patients with lung cancer.

\begin{tabular}{cccccc}
\hline Characteristic & No. $(\%)$ & CHR $\mathbf{( 9 5 \% ~ C I ) ~}$ & $p$-Value & AHR (95\% CI) & $p$-Value \\
\hline miR-1-3p & $(\boldsymbol{n}=\mathbf{4 3 3 )}$ & & & 1.00 & \\
\hline Low & $326(75.3)$ & 1.00 & & $0.54(0.31-0.94)$ & 0.029 \\
\hline High & $107(24.7)$ & $0.48(0.28-0.83)$ & 0.009 & 0.59 \\
\hline
\end{tabular}

Abbreviation: OS, Overall survival; CHR, crude hazard ratio; AHR, adjusted hazard ratio. AHR were adjusted for AJCC pathological stage (II, III and IV vs. I).

\section{Discussion}

Evidence of frequent overexpression of FAM83A in human cancers, including lung and breast cancer and pancreatic ductal adenocarcinoma, is increasing $[5,6,9,11,18]$. In a study by Shi et al., TCGA database analysis of LUAD and LUSC demonstrated a positive correlation between FAM83A and its antisense RNA FAM83A-AS1 [8]. As FAM83A-AS1 is located at the intron of FAM83A, both FAM83A and FAM $83 A-A S 1$ may be regulated through chromatin remodeling. To our best knowledge, until now, no studies have described the mechanism of FAM $83 A$ overexpression in human cancers. In a study by Li et al., bioinformatics analysis found increased FAM $83 A$ expression in patients with lung cancer who smoked compared with in those who did not, suggesting that cigarette smoking can induce FAM $83 \mathrm{~A}$ expression [19].

In this study, we reported for the first time that low miR-1-3p expression may contribute to FAM83A overexpression in human lung cancer. miR-1-3p was significantly downregulated in lung cancer tissues 
compared with in adjacent normal tissues. Previous study indicated that miR-1-3p is a conserved miRNA with high expression in the muscle tissues, but low abundant in normal lung tissues [20]. In present study, miR-1-3p was indicated to have higher expression in the corresponding adjacent normal tissue than LUAD by analyzing TCGA database. These results implied that miR-1-3p expression may be gradually induced when the lung tissue begins to develop precancerous lesions. However, the expression levels of miR-1-3p might be further reduced in a malignancy LUAD. This hypothesis needs more experiments to demonstrate it in the future. A study reported that ectopic expression of miR-1-3p in lung cancer cells suppresses proliferation, impairs cell cycle progression, and inhibits migration and invasion ability by silencing ANXA2 expression [21]. Jiao et al. demonstrated that miR-1-3p sensitizes hepatocyte growth factor-induced gefitinib-resistant lung cancer cells by modulating c-Met signaling [22]. These findings indicate that miR-1-3p suppresses tumor growth by regulating the growth and motility of lung cancer cells. FAM $83 A$ is a novel target gene for miR-1-3p in lung cancer, and FAM83A overexpression may result from downregulation of miR-1-3p.

Symptom nonspecificity and early-stage metastasis make early diagnosis of lung cancer difficult. To establish a prognostic risk model, therefore, numerous studies have investigated prognostic factors for lung cancer. Several recent studies reported that FAM83A was significantly overexpressed in lung cancer tissues compared with in adjacent normal tissues, and that this high expression were closely associated with poor prognosis of patients with lung cancer [7]. Zhang et al. studied the expression of 4 genes, MYO1E, ERO1L, C1QTNF6, and FAM83A, to build a prognostic panel for lung cancer. Functional enrichment analysis indicated that dysfunction in these genes are involved in regulation and progression of the cell cycle, synthesis and assembly of nucleic acids, and histone modification [23]. Lee et al. suggested a positive correlation of FAM83A with therapeutic resistance of tyrosine kinase inhibitors in breast cancer. FAM83A interacts with and causes phosphorylation of proto-oncogene c-RAF and PI3K p85 upstream of MAPK and downstream of the epidermal growth factor receptor [9]. The present study demonstrates that FAM83A expression may be a prognostic biomarker for pathological stage, lymph node metastasis, and overall survival of patients with lung cancer.

FAM83A overexpression promotes cell proliferation and metastasis in lung cancer through the modulation of cancer-related signaling pathways, including PI3K/ATK/Snail, Wnt, Hippo, and MAPK [24-27]. Studies have reported that choline kinase is overexpressed in lung cancer and has been demonstrated to play a critical role in the onset of human cancer [28,29]. We revealed that FAM $83 A$ knockdown-induced inhibition of lung cancer cell growth and motility was partially caused by the suppression of EGFR/MAPK/CHKA signaling activity. Jiao et al. reported that miR-1-3p expression reduced the phosphorylation of EGFR in lung cancer cells [22]. Similar results were observed for neck squamous carcinoma cells by using miR-1 to mimic transfection [30]. Furthermore, in silico analysis revealed that miR-1-3p may be targeted to the $3^{\prime}$ UTR of FAM83A. These findings suggest that the miR-1-FAM83A axis is critical in lung cancer cell growth and metastasis.

In summary, our findings demonstrated that FAM $83 A$ plays an oncogenic role in regulating lung cancer cell growth and motility via modulating EGFR/MAPK/CHKA signaling activity. In addition, we reported for the first time that miR-1-3p dysfunction may contribute to FAM83A overexpression and that high $F A M 83 A$ expression could be a biomarker for poor lung cancer prognosis. Our findings can serve as a reference for biomarker identification or therapeutic development for LUAD.

\section{Materials and Methods}

\subsection{Cell Culture}

Lung cancer cell lines A549 and H1299 were obtained from the American Type Culture Collection and sustained in Roswell Park Memorial Institute Medium 1640 supplemented with 10\% inactivated fetal bovine serum (Invitrogen, Carlsbad, CA, USA). Total RNA was isolated using TRIzol reagent (Invitrogen, Carlsbad, CA, USA) according to the manufacturer's instructions. The concentrations 
were measured on a Nanodrop 1000 spectrophotometer (Nanodrop Technologies, LLC, Wilmington, DE, USA).

\subsection{Gene Expression Profiles According to Cancer Genome Atlas Data}

The Cancer Genome Atlas (TCGA) program collects both cancerous and corresponding normal tissues from patients with LUAD and LUSC. We accessed downloaded TCGA data on RNA sequences in LUAD and LUSC tissues from the Genomic Data Commons Data Portal. The clinical information of patients with LUAD and LUSC was also downloaded. The expression profiles of 515 LUAD and 59 corresponding adjacent normal tissue samples as well as 501 LUSC and 49 corresponding adjacent normal tissue samples were obtained from the TCGA data portal. The transcriptome profiles of 485 patients with LUAD and 494 patients with LUSC were used to perform overall survival analysis using the Kaplan-Meier method. The clinical pathological stages were assessed using the eighth edition of the TNM staging system.

\subsection{Real-Time Reverse Transcription Polymerase Chain Reaction}

Total RNA was reverse transcribed using random primers and SuperScript III Reverse Transcriptase according to the manufacturer's instructions (Invitrogen). Complementary DNA was then subjected to gene expression analysis using Synergy Brands (SYBR) Green Master Mix (Applied Biosystems, Foster City, CA, USA) on the 7900 HT Fast Real-Time PCR System (Applied Biosystems). Glyceraldehyde 3-phosphate dehydrogenase (GAPDH) was used as an internal control. The sequences of all primers used are presented as follows:

GAPDH-F: TGCACCACCAACTGCTTAGC

GAPDH-R: GGCATGGACTGTGGTCATGAG

FAM83A-F: CCCTATAAAGAGTGGCAACAG

FAM83A-R: AACAGTGAGCAAACACACCG

\subsection{RNA Interference Knockdown of FAM83A}

H1355 or A549 cells were transfected with si-FAM83A\#1 and si-FAM83A\#2 oligonucleotides directed against FAM83A (Sigma, Billerica, MA, USA), respectively. Random sequence siRNA oligonucleotides (Sigma) were used as a negative control. After $48 \mathrm{~h}$ of transfection, FAM83A expression was confirmed through real-time reverse transcription polymerase chain reaction.

\subsection{Colony Formation Assay}

In total, 4000 cells with FAM83A knockdown were seeded in 6-well plates and then incubated at $37^{\circ} \mathrm{C}$ for 2 weeks. The colonies were fixed with $3.7 \%$ formaldehyde for $10 \mathrm{~min}$ and stained with crystal violet. Relative colony formation ability was then determined using a spectrophotometer at a wavelength of $620 \mathrm{~nm}$.

\subsection{Cell Proliferation, Migration, and Invasion Assay}

After the siFAM83A oligonucleotides were transfected into the lung cancer cells, a total of 5000 cells were seeded in 96-well plates. Cell proliferation was then measured at 0, 1, 2, 3 and 4 days using the CellTiter-Glo One Solution Assay (Promega Corporation, Madison, WI, USA).

\subsection{Cell Migration and Invasion Ability}

The migration and invasion abilities of the lung cancer cells with FAM83A knockdown were assessed in vitro in transwell chambers (Costar, Lowell, USA), as we previously described [31]. All experiments were repeated 3 times. 


\subsection{Microarray Data Analysis}

The total RNA sources used were as follows: (1) control \#1 cells, (2) control \#2 cells, (3) siFAM83A-\#1 cells, and (4) siFAM83A-\#2 cells. The complementary DNA probes were derived from paired RNA samples from the FAM83A knockdown cells or the control cells. The probes were labeled using Cy3-dCTP (green) or Cy5-dCTP (red) and then spotted onto microarray chips. The microarray experiments and data analysis were performed by Welgene Biotech (Taipei, Taiwan) using Agilent Oligo Chips. The differentially expressed genes (genes with FAM83A knockdown v.s. control with fold change $\geq 1.5$ and $\leq 0.75$ and $p<0.05$ ) were selected from the microarray data. These differentially expressed genes were subjected for gene ontology analysis using Database for Annotation, Visualization and Integrated Discovery (version 6.8) [32] to identify the significantly enriched pathways.

\subsection{Western Blotting}

The total cell lysates were extracted with the radioimmunoprecipitation assay buffer $(50 \mathrm{mM}$ Tris $\mathrm{HCl}, \mathrm{pH}$ 8.0, $150 \mathrm{mM} \mathrm{NaCl}, 1 \% \mathrm{NP}-40,0.5 \%$ deoxycholic acid, $0.1 \%$ sodium dodecyl sulfate). Total proteins were separated through electrophoresis in 6-10\% sodium dodecyl sulfate-polyacrylamide gel and transferred onto nitrocellulose filter membranes (Millipore, Billerica, MA, USA). The membranes were then incubated with a blocking buffer for $1 \mathrm{~h}$ at room temperature, and incubated with the primary antibodies overnight at $4{ }^{\circ} \mathrm{C}$. For a description of these antibodies, please refer to our previous study [33]. The membranes were then incubated with a horseradish peroxidase-conjugated secondary antibody for $1 \mathrm{~h}$ at room temperature to detect the primary antibody. In this study, the primary antibodies were used: CCNB1 (1:1000; 55004-1-AP, Proteintech Group, Inc., Rosemont, IL, USA), CCND1 (1:200; MA5-16356, Thermo Fisher Scientific Inc., Waltham, MA, USA), CDKN1B (p27) (1:500; 25614-1-AP, Proteintech), FAM83A (1:500, 20618-1-AP, Proteintech), EGFR (1:1000; \#4267, Cell Signaling Technology, Inc., Beverly, MA, USA), p-EGFR (1:1000; \#3777, Cell Signaling Technology, Inc.), MAPK (1:1000; \#9107, Cell Signaling Technology, Inc.), p-MAPK (1:1000; \#4370, Cell Signaling Technology, Inc.), CHKA (1:1000; \#13422, Cell Signaling Technology, Inc.), and Actin (ACTB) (1:5000; MAB1501, EMD Millipore, Billerica, MA, USA).

Finally, the proteins were visualized using the WesternBright ECL HRP substrate (Advansta Inc., San Jose, CA, USA) and captured using the BioSpectrum 500 Imaging System (UVP, LLC, Upland, CA, USA).

\subsection{Stem-Loop Reverse Transcription PCR}

According to the manufacturer's instructions (Invitrogen, Carlsbad, CA, USA) and as we described previously [34], total RNA was reverse transcribed through a stem-loop reverse transcription reaction by using miR-1-3p reverse transcription primers (5'-CTCAACTGGTGTCGTGGAGTCGG CAATTCAGTTGAGATACATAC-3') and SuperScript III Reverse Transcriptase. Gene expression was assessed using an SYBR Green I assay (Applied Biosystems) and the expression level of miR-1-3p was normalized to that of $\mathrm{U} 6(\Delta \mathrm{Ct}=\mathrm{miR}-1-3 \mathrm{p} \mathrm{Ct}-\mathrm{U} 6 \mathrm{Ct})$. The sequence of primers of real-time PCR was as follows:

miR-1-3p-GSF: 5'-CGGCGGTGGAATGTAAAGAAGT-3'

Universal reverse: $5^{\prime}$-CTGGTGTCGTGGAGTCGGCAATTC-3'

U6-F: 5'-CTCGCTTCGGCAGCACA-3'

U6-R: $5^{\prime}$-AACGCTTCACGAATTTGCGT-3

\subsection{1. miR-1-3p Mimics Trasfection}

A549 cells were transfected with $10 \mathrm{nM}$ of miRNA-1-3p mimics (sense: $5^{\prime}$-UGGAAUGUAAAGAA GUAUGUAU-3'; antisense: $5^{\prime}$-ACAUACUUCUUUACAUUCCAUU-3') or a random siRNA sequence as control (N.C) using Lipofectamine RNAiMAX reagent (13778150, Thermo Fisher Scientific Inc., Waltham, MA, USA). 


\subsection{2. miRNA Target Candidates and Luciferase Reporter Assay}

Identification of miRNA candidates for binding to $3^{\prime}$-UTR of FAM83A mRNA was done using the prediction tool on microRNA.org [35]. The $3^{\prime}$-UTR sequences and seed region mutant of FAM83A were cloned into a pMIR-REPROT vector (AM5795, Thermo Fisher Scientific). The pMIR-REPROT-FAM83A or pMIR-REPROT-FAM83A (mutant) vector was cotransfected with or without the miR-1-3p mimics into cells using Lipofectamine 2000 (Invitrogen, Thermo Fisher Scientific). After 24 h of transfection, the luciferase activity was examined using the Dual-Glo Luciferase Assay System (Promega Corporation, Madison, WI, USA). The detailed information was described in our previous study [34].

\subsection{Statistical Analysis}

The TCGA data for the expression levels of FAM83A or miR-1-3p in patients with LUAD were analyzed using Student $t$ tests. Cumulative survival curves were generated using the Kaplan-Meier method, and differences between survival curves were compared using the log-rank test. The correlations between FAM83A and miR-1-3p in LUAD tissues were analyzed using Pearson correlation. Cell proliferation, colony formation, migration and invasion experiments were performed in triplicate. The histograms present the mean values, and the error bars indicate the standard deviation. These data were analyzed using Student $t$ tests. The difference was considered to be significant when $p<0.05$.

Supplementary Materials: Supplementary Materials can be found at http://www.mdpi.com/1422-0067/21/22/ 8833/s1.

Author Contributions: P.-J.L., K.-W.T. and Y.-H.C. executed the study and drafted the manuscript. The TCGA database analysis was performed by K.-W.T. and Y.-T.T.; Y.-T.T., C.-Y.Y. (Chung-Yu Yeh) and H.-Y.Y. performed the in vitro cell biological assay. C.-Y.Y. (Chih-Yun Yang) supervised the study and edited the manuscript. All authors have read and agreed to the published version of the manuscript.

Funding: This work was supported by from the Kaohsiung Veterans General Hospital and the Kaohsiung Municipal Min-Sheng Hospital under grant numbers VGHKS108-007 and KMSH-10903, respectively.

Conflicts of Interest: The authors declare no conflict of interest.

\section{References}

1. Ferlay, J.; Shin, H.R.; Bray, F.; Forman, D.; Mathers, C.; Parkin, D.M. Estimates of worldwide burden of cancer in 2008: GLOBOCAN 2008. Int. J. Cancer 2010, 127, 2893-2917. [CrossRef] [PubMed]

2. Campbell, J.D.; Alexandrov, A.; Kim, J.; Wala, J.; Berger, A.H.; Pedamallu, C.S.; Shukla, S.A.; Guo, G.; Brooks, A.N.; Murray, B.A.; et al. Distinct patterns of somatic genome alterations in lung adenocarcinomas and squamous cell carcinomas. Nat. Genet. 2016, 48, 607-616. [CrossRef] [PubMed]

3. Socinski, M.A.; Crowell, R.; Hensing, T.E.; Langer, C.J.; Lilenbaum, R.; Sandler, A.B.; Morris, D.; American College of Chest, P. Treatment of non-small cell lung cancer, stage IV: ACCP evidence-based clinical practice guidelines (2nd edition). Chest 2007, 132, 277S-289S. [CrossRef] [PubMed]

4. Cipriano, R.; Miskimen, K.L.; Bryson, B.L.; Foy, C.R.; Bartel, C.A.; Jackson, M.W. Conserved oncogenic behavior of the FAM83 family regulates MAPK signaling in human cancer. Mol. Cancer Res. 2014, 12, 1156-1165. [CrossRef] [PubMed]

5. Chen, S.; Huang, J.; Liu, Z.; Liang, Q.; Zhang, N.; Jin, Y. FAM83A is amplified and promotes cancer stem cell-like traits and chemoresistance in pancreatic cancer. Oncogenesis 2017, 6, e300. [CrossRef]

6. Parameswaran, N.; Bartel, C.A.; Hernandez-Sanchez, W.; Miskimen, K.L.; Smigiel, J.M.; Khalil, A.M.; Jackson, M.W. A FAM83A Positive Feed-back Loop Drives Survival and Tumorigenicity of Pancreatic Ductal Adenocarcinomas. Sci. Rep. 2019, 9, 13396. [CrossRef]

7. Richtmann, S.; Wilkens, D.; Warth, A.; Lasitschka, F.; Winter, H.; Christopoulos, P.; Herth, F.J.F.; Muley, T.; Meister, M.; Schneider, M.A. FAM83A and FAM83B as Prognostic Biomarkers and Potential New Therapeutic Targets in NSCLC. Cancers 2019, 11, 652. [CrossRef]

8. Shi, R.; Jiao, Z.; Yu, A.; Wang, T. Long noncoding antisense RNA FAM83A-AS1 promotes lung cancer cell progression by increasing FAM83A. J. Cell. Biochem. 2019, 120, 10505-10512. [CrossRef] 
9. Lee, S.Y.; Meier, R.; Furuta, S.; Lenburg, M.E.; Kenny, P.A.; Xu, R.; Bissell, M.J. FAM83A confers EGFR-TKI resistance in breast cancer cells and in mice. J. Clin. Investig. 2012, 122, 3211-3220. [CrossRef]

10. Bartel, C.A.; Jackson, M.W. HER2-positive breast cancer cells expressing elevated FAM83A are sensitive to FAM83A loss. PLoS ONE 2017, 12, e0176778. [CrossRef]

11. Liu, C.; Peng, X.; Li, Y.; Liu, S.; Hou, R.; Zhang, Y.; Zuo, S.; Liu, Z.; Luo, R.; Li, L.; et al. Positive feedback loop of FAM83A/PI3K/AKT/c-Jun induces migration, invasion and metastasis in hepatocellular carcinoma. Biomed. Pharmacother. 2020, 123, 109780. [CrossRef] [PubMed]

12. Grant, S. FAM83A and FAM83B: Candidate oncogenes and TKI resistance mediators. J. Clin. Investig. 2012, 122, 3048-3051. [CrossRef] [PubMed]

13. Cipriano, R.; Graham, J.; Miskimen, K.L.; Bryson, B.L.; Bruntz, R.C.; Scott, S.A.; Brown, H.A.; Stark, G.R.; Jackson, M.W. FAM83B mediates EGFR- and RAS-driven oncogenic transformation. J. Clin. Investig. 2012, 122, 3197-3210. [CrossRef] [PubMed]

14. McHugh, B.J.; Murdoch, A.; Haslett, C.; Sethi, T. Loss of the integrin-activating transmembrane protein Fam38A (Piezo1) promotes a switch to a reduced integrin-dependent mode of cell migration. PLoS ONE 2012, 7, e40346. [CrossRef]

15. Yang, X.N.; Lu, Y.P.; Liu, J.J.; Huang, J.K.; Liu, Y.P.; Xiao, C.X.; Jazag, A.; Ren, J.L.; Guleng, B. Piezo1 is as a novel trefoil factor family 1 binding protein that promotes gastric cancer cell mobility in vitro. Dig. Dis. Sci. 2014, 59, 1428-1435. [CrossRef]

16. Etem, E.O.; Ceylan, G.G.; Ozaydin, S.; Ceylan, C.; Ozercan, I.; Kuloglu, T. The increased expression of Piezo1 and Piezo2 ion channels in human and mouse bladder carcinoma. Adv. Clin. Exp. Med. Off. Organ Wroc. Med Univ. 2018, 27, 1025-1031. [CrossRef]

17. Zhang, J.; Zhou, Y.; Huang, T.; Wu, F.; Liu, L.; Kwan, J.S.H.; Cheng, A.S.L.; Yu, J.; To, K.F.; Kang, W. PIEZO1 functions as a potential oncogene by promoting cell proliferation and migration in gastric carcinogenesis. Mol. Carcinog. 2018, 57, 1144-1155. [CrossRef]

18. Zhang, J.T.; Lin, Y.C.; Xiao, B.F.; Yu, B.T. Overexpression of Family with Sequence Similarity 83, Member A (FAM83A) Predicts Poor Clinical Outcomes in Lung Adenocarcinoma. Med. Sci. Monit. Int. Med. J. Exp. Clin. Res. 2019, 25, 4264-4272. [CrossRef]

19. Li, Y.; Xiao, X.; Ji, X.; Liu, B.; Amos, C.I. RNA-seq analysis of lung adenocarcinomas reveals different gene expression profiles between smoking and nonsmoking patients. Tumour Biol. J. Int. Soc. Oncodev. Biol. Med. 2015, 36, 8993-9003. [CrossRef]

20. Ludwig, N.; Leidinger, P.; Becker, K.; Backes, C.; Fehlmann, T.; Pallasch, C.; Rheinheimer, S.; Meder, B.; Stahler, C.; Meese, E.; et al. Distribution of miRNA expression across human tissues. Nucleic Acids Res. 2016, 44, 3865-3877. [CrossRef]

21. Wang, Y.; Luo, X.; Liu, Y.; Han, G.; Sun, D. Long noncoding RNA RMRP promotes proliferation and invasion via targeting miR-1-3p in non-small-cell lung cancer. J. Cell. Biochem. 2019, 120, 15170-15181. [CrossRef] [PubMed]

22. Jiao, D.; Chen, J.; Li, Y.; Tang, X.; Wang, J.; Xu, W.; Song, J.; Li, Y.; Tao, H.; Chen, Q. miR-1-3p and miR-206 sensitizes HGF-induced gefitinib-resistant human lung cancer cells through inhibition of c-Met signalling and EMT. J. Cell Mol. Med. 2018, 22, 3526-3536. [CrossRef] [PubMed]

23. Zhang, W.; Shen, Y.; Feng, G. Predicting the survival of patients with lung adenocarcinoma using a four-gene prognosis risk model. Oncol. Lett. 2019, 18, 535-544. [CrossRef] [PubMed]

24. Zhou, F.; Geng, J.; Xu, S.; Meng, Q.; Chen, K.; Liu, F.; Yang, F.; Pan, B.; Yu, Y. FAM83A signaling induces epithelial-mesenchymal transition by the PI3K/AKT/Snail pathway in NSCLC. Aging 2019, 11, 6069-6088. [CrossRef] [PubMed]

25. Wang, Y.; Xu, R.; Zhang, D.; Lu, T.; Yu, W.; Wo, Y.; Liu, A.; Sui, T.; Cui, J.; Qin, Y.; et al. Circ-ZKSCAN1 regulates FAM83A expression and inactivates MAPK signaling by targeting miR-330-5p to promote non-small cell lung cancer progression. Transl. Lung Cancer Res. 2019, 8, 862-875. [CrossRef] [PubMed]

26. Liu, L.; Liao, G.Q.; He, P.; Zhu, H.; Liu, P.H.; Qu, Y.M.; Song, X.M.; Xu, Q.W.; Gao, Q.; Zhang, Y.; et al. Detection of circulating cancer cells in lung cancer patients with a panel of marker genes. Biochem. Biophys. Res. Commun. 2008, 372, 756-760. [CrossRef] [PubMed]

27. Zheng, Y.W.; Li, Z.H.; Lei, L.; Liu, C.C.; Wang, Z.; Fei, L.R.; Yang, M.Q.; Huang, W.J.; Xu, H.T. FAM83A Promotes Lung Cancer Progression by Regulating the Wnt and Hippo Signaling Pathways and Indicates Poor Prognosis. Front. Oncol. 2020, 10, 180. [CrossRef] 
28. Ramirez de Molina, A.; Gallego-Ortega, D.; Sarmentero, J.; Banez-Coronel, M.; Martin-Cantalejo, Y.; Lacal, J.C. Choline kinase is a novel oncogene that potentiates RhoA-induced carcinogenesis. Cancer Res. 2005, 65, 5647-5653. [CrossRef]

29. Ramirez de Molina, A.; Rodriguez-Gonzalez, A.; Gutierrez, R.; Martinez-Pineiro, L.; Sanchez, J.; Bonilla, F.; Rosell, R.; Lacal, J. Overexpression of choline kinase is a frequent feature in human tumor-derived cell lines and in lung, prostate, and colorectal human cancers. Biochem. Biophys. Res. Commun. 2002, 296, 580-583. [CrossRef]

30. Koshizuka, K.; Hanazawa, T.; Fukumoto, I.; Kikkawa, N.; Matsushita, R.; Mataki, H.; Mizuno, K.; Okamoto, Y.; Seki, N. Dual-receptor (EGFR and c-MET) inhibition by tumor-suppressive miR-1 and miR-206 in head and neck squamous cell carcinoma. J. Hum. Genet. 2017, 62, 113-121. [CrossRef]

31. Lin, M.H.; Chen, Y.Z.; Lee, M.Y.; Weng, K.P.; Chang, H.T.; Yu, S.Y.; Dong, B.J.; Kuo, F.R.; Hung, L.T.; Liu, L.F.; et al. Comprehensive identification of microRNA arm selection preference in lung cancer: miR-324-5p and -3p serve oncogenic functions in lung cancer. Oncol. Lett. 2018, 15, 9818-9826. [CrossRef] [PubMed]

32. Huang da, W.; Sherman, B.T.; Lempicki, R.A. Systematic and integrative analysis of large gene lists using DAVID bioinformatics resources. Nat. Protoc. 2009, 4, 44-57. [CrossRef] [PubMed]

33. Tsai, K.W.; Lo, Y.H.; Liu, H.; Yeh, C.Y.; Chen, Y.Z.; Hsu, C.W.; Chen, W.S.; Wang, J.H. Linc00659, a long noncoding RNA, acts as novel oncogene in regulating cancer cell growth in colorectal cancer. Mol. Cancer 2018, 17, 72. [CrossRef] [PubMed]

34. Chen, Y.C.; Wu, C.C.; Tu, Y.T.; Chen, Y.R.; Lee, M.C.; Tsai, K.W. Involvement of the MicroRNA-1-LITAF Axis in Gastric Cancer Cell Growth and Invasion. Anticancer Res. 2020, 40, 6247-6256. [CrossRef] [PubMed]

35. Betel, D.; Wilson, M.; Gabow, A.; Marks, D.S.; Sander, C. The microRNA.org resource: Targets and expression. Nucleic Acids Res. 2008, 36, D149-D153. [CrossRef] [PubMed]

Publisher's Note: MDPI stays neutral with regard to jurisdictional claims in published maps and institutional affiliations. 